\title{
Magnetic Resonance Imaging or Ultrasound in Localized Intermediate- or High-Risk Soft Tissue Tumors of the Extremities (MUSTT): Final Results of a Prospective Comparative Trial
}

\author{
Bianca Bignotti ${ }^{1,2}$, Federica Rossi ${ }^{2,3}$, Alessio Signori ${ }^{4}$, Nicola Solari ${ }^{1}$, Bruno Spina ${ }^{1}$, Carlo Martinoli ${ }^{1,4, *}$ \\ and Alberto Stefano Tagliafico ${ }^{1,4, *}$
}

check for

updates

Citation: Bignotti, B.; Rossi, F.; Signori, A.; Solari, N.; Spina, B.; Martinoli, C.; Tagliafico, A.S. Magnetic Resonance Imaging or Ultrasound in Localized

Intermediate- or High-Risk Soft Tissue Tumors of the Extremities (MUSTT): Final Results of a Prospective Comparative Trial. Diagnostics 2022, 12, 411. https://doi.org/10.3390/ diagnostics12020411

Academic Editors: Manuel Scimeca, Alessandro Mauriello, Rita Bonfiglio and Nicola Fusco

Received: 17 January 2022

Accepted: 28 January 2022

Published: 5 February 2022

Publisher's Note: MDPI stays neutral with regard to jurisdictional claims in published maps and institutional affiliations.

Copyright: (c) 2022 by the authors. Licensee MDPI, Basel, Switzerland. This article is an open access article distributed under the terms and conditions of the Creative Commons Attribution (CC BY) license (https:/ / creativecommons.org/licenses/by/ $4.0 /)$.
1 Ospedale Policlinico San Martino, 16132 Genoa, Italy; bianca.bignotti@hsanmartino.it (B.B.); nicola.solari@hsanmartino.it (N.S.); bruno.spina@hsanmartino.it (B.S.)

2 Department of Experimental Medicine (DIMES), University of Genoa, 16132 Genoa, Italy; federica.rossi@asl2.liguria.it

3 Ospedale Santa Corona, Pietra Ligure, 17027 Genoa, Italy

4 Department of Health Sciences (DISSAL), University of Genoa, 16132 Genoa, Italy; alessio.signori@unige.it

* Correspondence: carlo.martinoli@unige.it (C.M.); alberto.tagliafico@unige.it or albertotagliafico@gmail.com (A.S.T.)

\begin{abstract}
Objectives: To report final results of the MUSTT trial, which has been designed to independently compare magnetic resonance imaging (MRI) and ultrasound (US) for local recurrences of non-metastatic patients operated for malignant soft tissue tumors (STT). Methods: Magnetic resonance imaging or ultrasound in soft tissue tumors (MUSTT) is a prospective monocentric study recruiting asymptomatic, non-metastatic patients operated on for localized soft tissue sarcomas between 2015 and April. Eligible patients had MRI and physician-performed ultrasound (US) with an independent interpretation of imaging. Outcome measures were compared using ROC analysis and the $\mathrm{X}^{2}$ test. An analysis of all patients was performed on a per-follow-up event basis. Results: A total of $n=51$ patients who met the inclusion criteria agreed to participate. Among them, $n=8$ were lost to follow-up, $\mathrm{n}=6$ had US and MRI acquired after a time frame $>7$ days and were therefore excluded. Complete data available for 37 patients with 232 MRI and 232 US scan were finally considered (men/women: 18/20; age range, 18-84 years). Recurrences within 5 years occurred in 10/37 patients (27\%). ROC analysis comparing US and MRI showed an AUC with $95 \%$ confidence intervals of 0.909 (0.832 to 0.981) for US and 0.966 (0.939 to 0.989) for MRI with Prob $>X^{2}=0$. Conclusions: Each of these tests detected local recurrences with suitable accuracy. MRI did not result clearly superior to US in terms of diagnostic accuracy, but US showed some false positive or negative results.
\end{abstract}

Keywords: sarcoma; ultrasound; magnetic resonance imaging; accuracy; recurrence

\section{Introduction}

Soft tissue tumors (STT) are represented by a wide range of different histological and molecular subsets with very low incidence populations at all ages [1]. STT represents the majority of sarcomas (soft tissue tumors $\sim 75 \%$, gastrointestinal $\sim 15 \%$, and $\sim 10 \%$ bone sarcomas) [2]. The prognosis of STT is influenced by different factors such as grading, resection margin, location, age $>64$ years, and distant sarcoma metastasis [1]. However, local recurrences (LRs) not only influence tumor control locally but also result to influence overall survival in several investigations [3-5]. The rate of LRs has been reported to vary from $8.5 \%$ after 24 months to $20-32 \%$ after 10 years [6]. The risk of LRs is higher in the first years after surgery, and it has been estimated that $60 \%$ to $70 \%$ of recurrences occur within 2 years and $>90 \%$ occur by 5 years [7]. Radiotherapy reduces LRs rates [8]. Although some studies showed that the survival rate of patients with STT, especially those with 
high-risk lesions, could be improved by consistent local follow-ups with imaging, there are not currently accepted evidence-based consensus guidelines on how and to what extent regular follow-up imaging influences the outcome of these patients [9]. In standard radiological clinical practice, both ultrasound (US) and magnetic resonance imaging (MRI) are currently used to rule out a recurrence in patients operated on for STT [1,2,10-12]. It is widely accepted that US is highly accessible, radiation-free, easily repeatable, fast, and with a spatial resolution higher than MRI when very-high-frequency transducers evaluate superficial tissues [10]. According to guidelines, STTs should be managed in tertiary sarcoma centers; however, radiologists can be involved in follow-up strategies even outside tertiary centers for practical reasons [2,10,12]. Following 2018 ESMO (European Society of Medical Oncology) guidelines on follow-up imaging of STT, there is no clear evidence to indicate the optimal routine follow-up of surgically treated patients with localized disease nor on the use of US and/or MRI or CT to detect LRs [2]. Recent retrospective studies found encouraging results regarding the performance of US in the detection of LRs in patients with localized soft tissue sarcomas of the limb, which resulted in having $88 \%$ sensitivity and $94 \%$ specificity [13]. However, there is a need for prospective studies because a practical approach is still in place at several institutions with frequent follow-up even every 3-4 months in the first $2-3$ years for intermediate-high-risk lesions $[2,10,12]$. Therefore, considering that performing US is easier and undoubtedly less stressful for patients than having a high-field MR (1.5T or 3.0T as suggested by guidelines), the purpose of the study was to prospectively compare MRI and US in the detection of local recurrences of adults patients with localized intermediate/high-risk soft tissue tumors of the limbs.

\section{Materials and Methods}

Magnetic resonance imaging or ultrasound in soft tissue tumors (MUSTT) was implemented as a prospective monocentric study recruiting asymptomatic, non-metastatic patients operated on for localized soft tissue sarcomas. Our aim was to compare the detection of LRs using widely adopted imaging methods such as US and MRI that allow independent test interpretation. The study received IRB approval (304REG2015), and the patients involved provided written informed consent. MUSTT is a registered study (ClinicalTrials.gov Identifier: NCT02834585) sponsored by the University of Blind City for Review and by the Blinded for Review.

\subsection{Patients}

Patients with STT who met the inclusion criteria were prospectively included and studied with US and MRI in our center, which is a regional referral center for diagnosis and treatment of STT and followed-up every 3-4 months. Patients to be included had to meet the following inclusion criteria:

- $\quad$ Age > 18 years;

- $\quad$ Surgery for proven primary intermediate or high-risk STT of the upper or lower limb (e.g., spindle-cell or pleomorphic histology, myxoid-round cell liposarcoma, leiomyosarcoma, synovial sarcoma, malignant peripheral nerve sheath tumor, undifferentiated pleomorphic sarcoma, myxofibrosarcoma, pleomorphic liposarcoma, pleomorphic rhabdomyosarcoma, et al.) according to WHO criteria;

- $\quad$ Localized disease (non-metastatic);

- $\quad$ ECOG performance status $<1$;

- $\quad$ Signed informed consent;

- $\quad$ Complete US and MRI follow-up every 3-4 months;

- $\quad$ Follow-up of 24 months for negative US and MRI findings;

- Time-lapse between US and MRI $<7$ days.

Exclusion criteria were the following:

- Pregnancy or lactation;

- Other malignancies within the past 5 years;

- Metastatic disease; 
- Low-risk sarcomas;

- $\quad$ Lack of informed consent or limited compliance;

- Impossibility of warranting imaging follow-up at the center;

- Absence of definitive histological diagnosis on surgically excised masses (when surgery was not performed or performed in other centers).

\subsection{Imaging Follow-Up Program}

The post-operative to detect LRs imaging was planned with US and MR imaging every 4 months for the first 5 years and follow-up of 24 months for negative US and MRI findings after 5 years. Clinical assessment, chest CT and relevant assessment were performed to exclude metastatic disease as suggested by current protocols [2].

\subsection{Ultrasound}

All US examinations of upper and lower limbs were performed by a team of 4 experienced radiologists (minimum experience: 6 years; maximum experience 27 years) with specific expertise in musculoskeletal imaging and soft-tissue masses as suggested by the Tumor Subcommittee of the European Society of Musculoskeletal Radiology (ESSR) [10,14]. Ultrasound probes for limb evaluation to rule out the presence of LRs included lastgeneration broadband linear array transducers (at least $13 \mathrm{MHz}$ of frequency) of different vendors (Esaote My Lab, different versions, and Canon Aplio 800). Standard US evaluation included in the radiological report: anatomical location of the lesion with a clear description of anatomical relationships among the lesion, the muscles, the nerves, and the vessels, intra- or intermuscular location and compartmental involvement; size, pattern of growth, relation to the fascia (superficial or deep), color-Doppler evaluation, presence or absence of intra-lesion necrosis, bleeding, posterior acoustic enhancement/shadowing, suspected calcifications, shape, borders/margins. Finally, the radiologist had to conclude if the US examination was consistent with LR or not on the basis of ESSR criteria and personal judgment [10]. In the case of possible LR at US, further biopsy was warranted.

\subsection{Magnetic Resonance Imaging}

Standard musculoskeletal limb MRI was performed on different vendor 1.5T (Siemens Magentom Aera 1.5T or Magnetom Avanto1.5T) or 3T scanners (Siemens MAGNETOM Prisma 3.0T) with anatomical T1-weighted sequences, T2-weighted sequences with and without fat saturation, and T1-weighted sequences with fat saturation before and after intravenous administration of gadolinium chelates as per ACR guidelines (https: / /www. acr.org / accessed on 17 June 2021). MRI planes and coils were tailored on the anatomical region with at least $4 \mathrm{~mm}$ of slice thickness [15-17]. Coils used were: 4-channel flex coils of different sizes for the extremities combined, when necessary, with 8- to 32-channel phased-array body coils included and Tx/Rx Knee 15 Flare Coil for the knees. The MRI protocol started from the following parameters slightly adapted to the region considered: slice thickness at least $4 \mathrm{~mm}$, slice spacing $=1 \mathrm{~mm}$, matrix size $384 x \mathrm{~T} 1$-weighted MR imaging repetition time/echo time (TR/TE) $500 \mathrm{~ms} / 8 \mathrm{~ms}$; acquisition voxel size $\left(\mathrm{mm}^{3}\right)$ $0.6 \times 0.7 \times 3.0$; T2-weighted MR imaging TR/TE $6200 \mathrm{~ms} / 110 \mathrm{~ms}$; acquisition voxel size $\left(\mathrm{mm}^{3}\right) 0.6 \times 0.7 \times 3.0 ;$ T1-weighted MR imaging with gadolinium TR/TE = 500/12 ms; acquisition voxel size $\left(\mathrm{mm}^{3}\right) 0.6 \times 0.7 \times 3.0$; T2-weighted MR imaging and T1-weighted MR imaging with gadolinium is acquired with fat saturation. As for US, the radiologist had to state if MR was consistent with LR or not according to current guidelines [10]. Each patient included at every follow-up had both US and MRI acquired and reported within 7 days by different radiologists independently. Radiologists were blinded to the US findings if they were reporting the MRI and vice versa. The radiologist who performed US did not perform MRI and vice versa to guarantee independent reading of US and MRI. Radiological findings included in the MRI report to detect recurrences: location, three-dimensional size, morphology, shape, borders, relation to the superficial fascia, intra- extra- compartmental location, relation to adjacent structures (vessels, nerves, joints, ... ), and surrounding 
tissue alterations, distance to an external landmark, satellites, multiplicity, loco-regional lymph nodes. The signal intensity of the lesions (e.g., presence of fat, hypo-intensity on fluid-sensitive sequences), homogeneity (esp. heterogeneity of $>50 \%$ of the tumor volume on fluid-sensitive fat-suppressed images), diffusion restriction (if available, with ADC), vascularity, and enhancement. The presence of a possible recurrence was defined according to ESSR guidelines [10] and then confirmed or excluded by biopsy.

To assess the diagnostic performance of US and MR on a per-event and per-lesion basis, histology and surgery were used as a reference standard for US, and MRI reported as positive (true positive). The false positive for US or MRI was defined when histology was negative after a positive imaging evaluation. The US or MRI false negative was assigned if LRs appeared before the next scheduled follow-up following literature suggestions [4,18]. No indeterminate US and MRI reports were allowed for the purpose of this study.

\subsection{Statistical Analysis}

US and MRI reports were compared against the reference standard (histology, surgery, or follow-up) on a per-even and per-lesion basis for each follow-up event. ROC curves for each modality and in combination were calculated. In addition, sensitivity and specificity with $95 \%$ confidence intervals (CIs) were estimated. ROC curves and standard diagnostic performance indicators were also estimated (positive predictive value (PPV), negative predictive value (NPV), the likelihood ratio for positive results ( $\mathrm{LH}+$ ), accuracy, and posttest probability (post-P). A p-value $<0.05$ (2-sided) was considered statistically significant. Logistic multiple regression was performed to exclude or identify the effect of independent variables (e.g., patient age, sex, reporting radiologist) on diagnostic accuracy. Commercially available software (MedCalc Software Ltd. Diagnostic test evaluation calculator. https: / / www.medcalc.org/calc/diagnostic_test.php (Version 20.009; accessed 2 July 2021, SPSS version 14, Chicago, Ill and STATA 17) were used for analysis.

\subsection{Power Analysis}

Power analysis and sample size calculation were performed considering a $1 \%$ difference in the incidence of the lesion between US and MRI. Indeed, an incidence of the event (recurrences) of 21/232 (9\%) and 24/232 (10\%) for US and MRI was present. The two study groups were considered to receive independent imaging evaluation with a binomial primary endpoint (LR or not). Considering an enrolment ratio of 1 (equal enrolment for US and MRI), the desired alpha (probability of type I error) of 0.05 , beta (type II error) of 0.2 , and a power (1-beta) of 0.8 , a sample size of $n=12,208$ was calculated to have a significant difference among two groups. This number has been considered clinically not relevant; therefore, this study was considered sufficiently powered [15-17,19,20].

\section{Results}

A total of $n=51$ patients who met the inclusion criteria agreed to participate. Among them, $\mathrm{n}=8$ were lost to follow-up, $\mathrm{n}=6$ had US and MRI acquired after a time frame $>7$ days and were therefore excluded. Complete data available for 37 patients with $232 \mathrm{MRI}$ and 232 US scans were finally considered for the analysis. From 37 patients with the complete US and MR imaging follow-up examination (men/women: 18/20; age range, 18-84 years), histology and clinical characteristics are reported in Table 1. After surgery for the primary tumor resection, margins were declared clear (R0) by the surgical team in $20 / 37$ (54\%), whereas in $17 / 37$ patients (46\%), microscopically involved margins (R1) were registered. Recurrences within 5 years occurred in $10 / 37$ patients (27\%): $n=4$ liposarcomas, $\mathrm{n}=4$ sarcomas, 2 synovial, 2 myxoid, $\mathrm{n}=1$ leiomyosarcoma, $\mathrm{n}=1$ atypical adipose tumor.

The median number of follow-up events per patient was 8 (range 6-24). Examples of US and MRI cases are illustrated in Figures 1-5. ROC analysis comparing US and MRI showed an AUC with 95\% confidence intervals of 0.909 (0.832 to 0.981$)$ for US and 0.966 (0.939 to 0.989 ) for MRI with $X^{2}=0.0766$ (Figure 6). Details for diagnostic performance of US and MRI are reported in Table 2. 
Table 1. Summary of patient's characteristics.

\begin{tabular}{|c|c|}
\hline & All Patients $(n=37)$ \\
\hline Age at diagnosis, years (median (range)) & $55(18-86)$ \\
\hline $\begin{array}{c}\text { Sex } \\
\text { Female }\end{array}$ & 19 \\
\hline Male & 18 \\
\hline Primary tumor size, mm (median (range)) & $55(7-200)$ \\
\hline Positive/negative resection margins (R1/R0) & $17 / 20$ \\
\hline Recurrence tumor size mm (median (range)) & $23(7-53)$ \\
\hline Number of patients with recurrences within 5 years & 10 \\
\hline Number of patients with multiple recurrences & 6 \\
\hline $\begin{array}{c}\text { Primary tumor location } \\
\text { Upper limb }\end{array}$ & 27 \\
\hline Lower limb & 10 \\
\hline Superficial location to superficial fascia of the recurrence & 3 \\
\hline Deep location to superficial fascia of the recurrence & 7 \\
\hline $\begin{array}{l}\text { Histologic subtypes } \\
\text { Hitection }\end{array}$ & \\
\hline Synovial sarcoma & 8 \\
\hline Nerve sheath tumors ${ }^{\circ}$ & 3 \\
\hline Myxofibrosarcoma & 6 \\
\hline Leiomyosarcoma & 4 \\
\hline Myxoid liposarcoma & 6 \\
\hline Pleomorphic sarcoma & 4 \\
\hline Other * & 6 \\
\hline
\end{tabular}

${ }^{\circ}$ MPNST: malignant peripheral nerve sheath tumor; Other *: 1(angiosarcoma epithelioid); 2(atypical adipose tumor); 1(extraskeletal osteogenic sarcoma); 1(angiomatoid fibrous histiocytoma); 1(hemangiopericytoma).

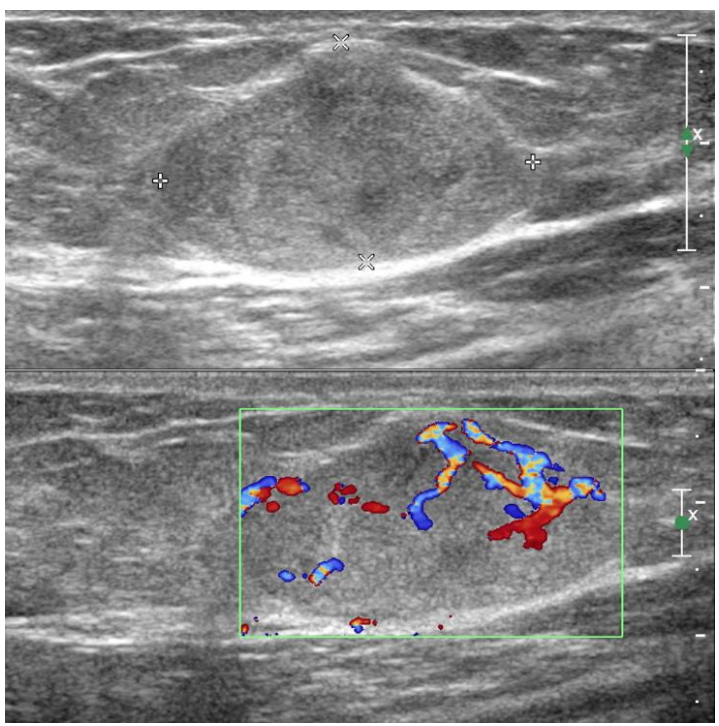

(a)

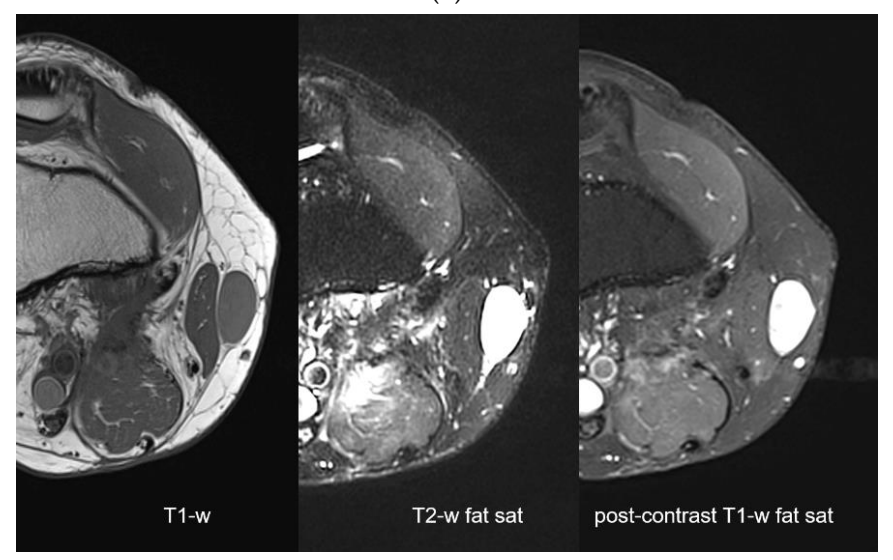

(b)

Figure 1. A 38-year-old man operated for a localized liposarcoma of the popliteal fossa. Images obtained after one year show a recurrence. Ultrasound images obtained at the medial aspect of the knee show a hyperechoic nodule (a) with intralesional color-Doppler signals and some slightly hypoechoic intralesional areas. MRI images (b) show a nodule isointense on T1-weighted sequences and hyperintense on both T2-weighted sequences with fat saturation and post-contrast T1-weighted sequences. Both US and MRI were reported as recurrence. 


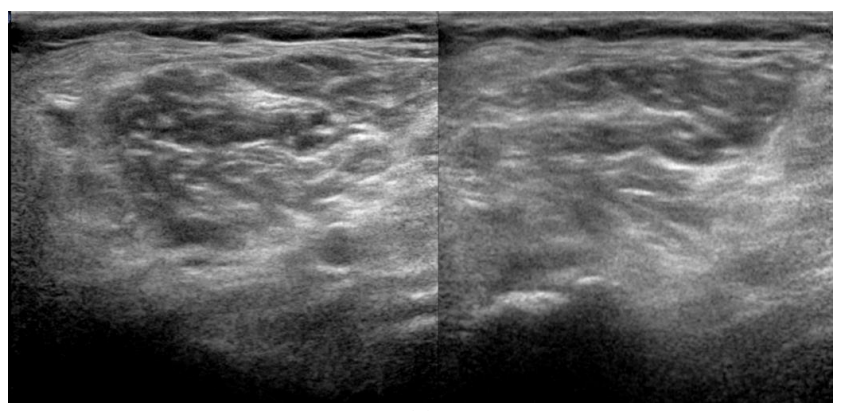

(a)

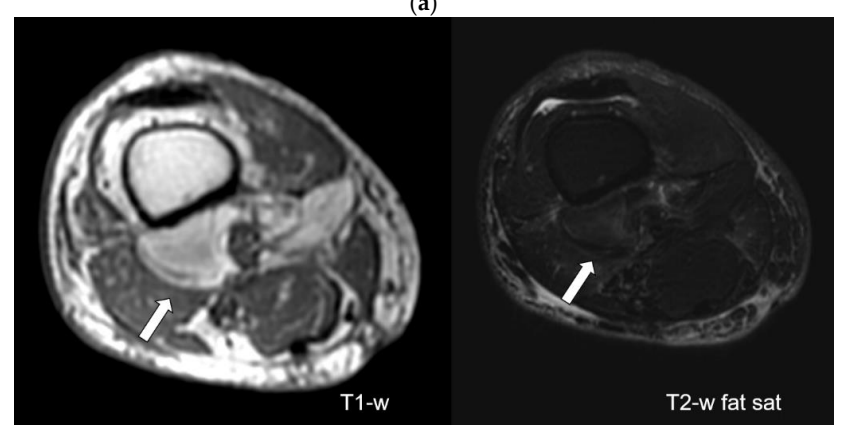

(b)

Figure 2. A 78-year-old man operated for a localized liposarcoma of the popliteal fossa in follow-up ultrasound images (a) obtained at the popliteal fossa were reported as negative for local recurrence with no recurrence demonstrated in the follow-up. MRI images (b) show a deep-located (white arrow) nodular lesion with MRI signal consistent with fat on T1-weighted sequences and T2-weighted sequences with fat saturation. A slight hyperintensity on T2-weighted sequences with fat saturation was present. In this case, ultrasound was reported negative for recurrence, whereas MRI was reported positive.

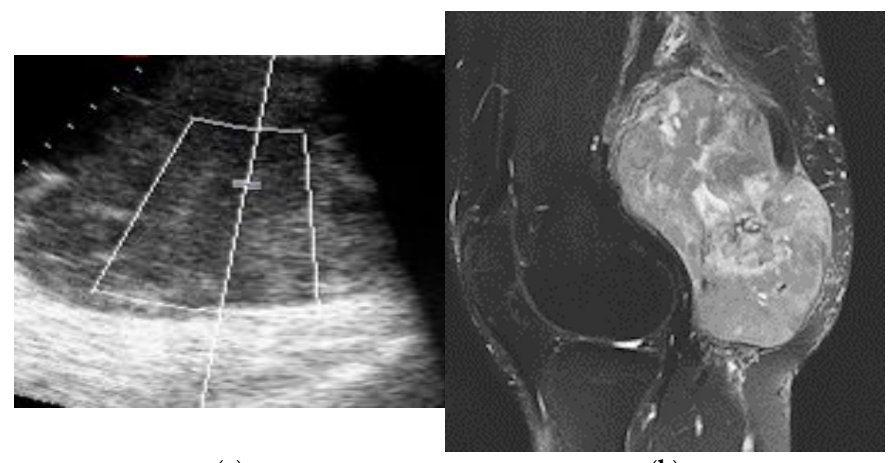

(a)

(b)

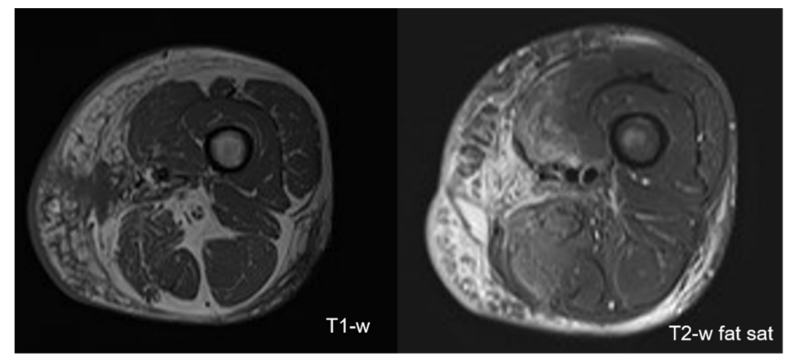

(c)

Figure 3. A 45-year-old man operated for a large MPNST with translocation $t(X ; 18)$ (p 11.2; q 11.2) and of genes SYT/SSX of the popliteal fossa shown by both US (a) and MRI (b). Follow-up did not show any changes consistent with relapse; only minor edema and fluid collection were present (c). Both MRI and US were reported negative for recurrence. 


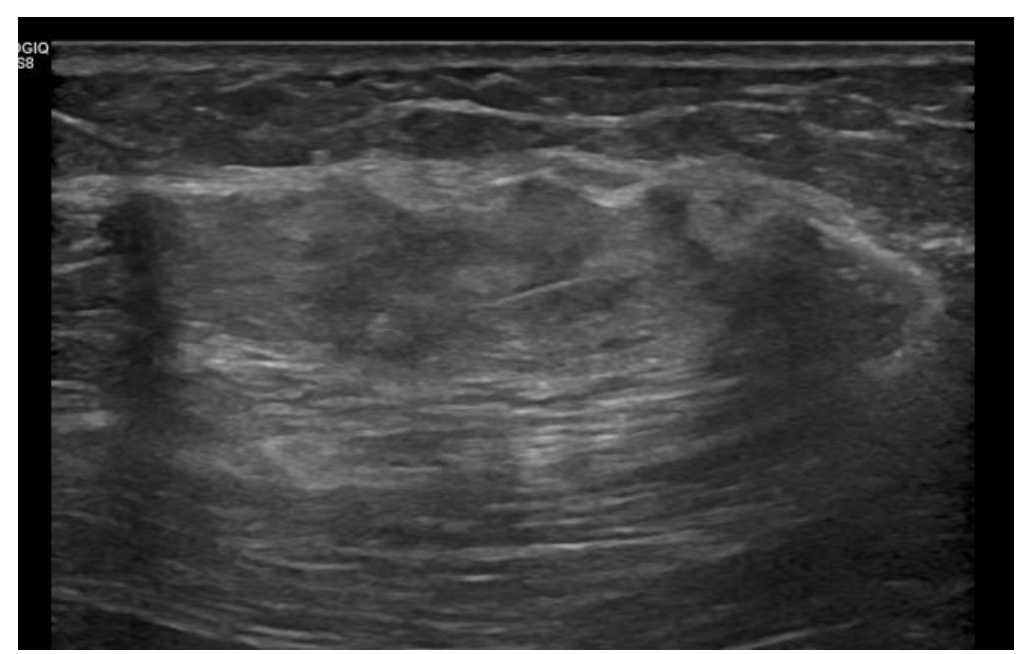

(a)

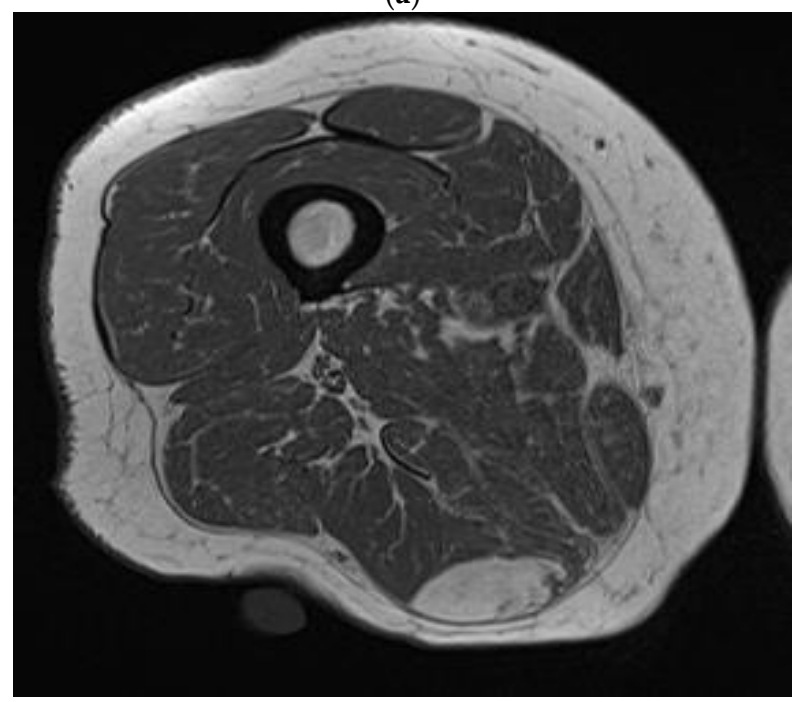

(b)

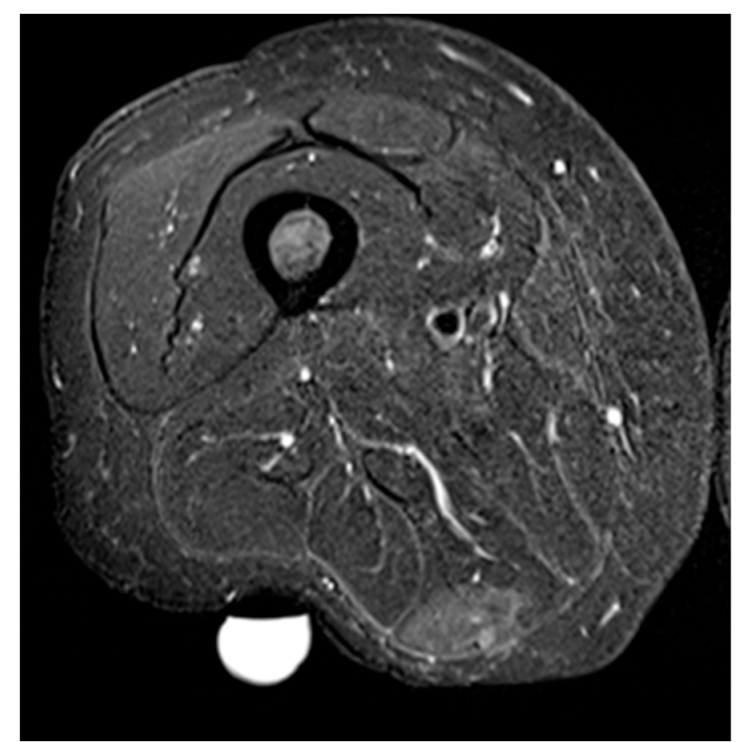

(c)

Figure 4. Cont. 


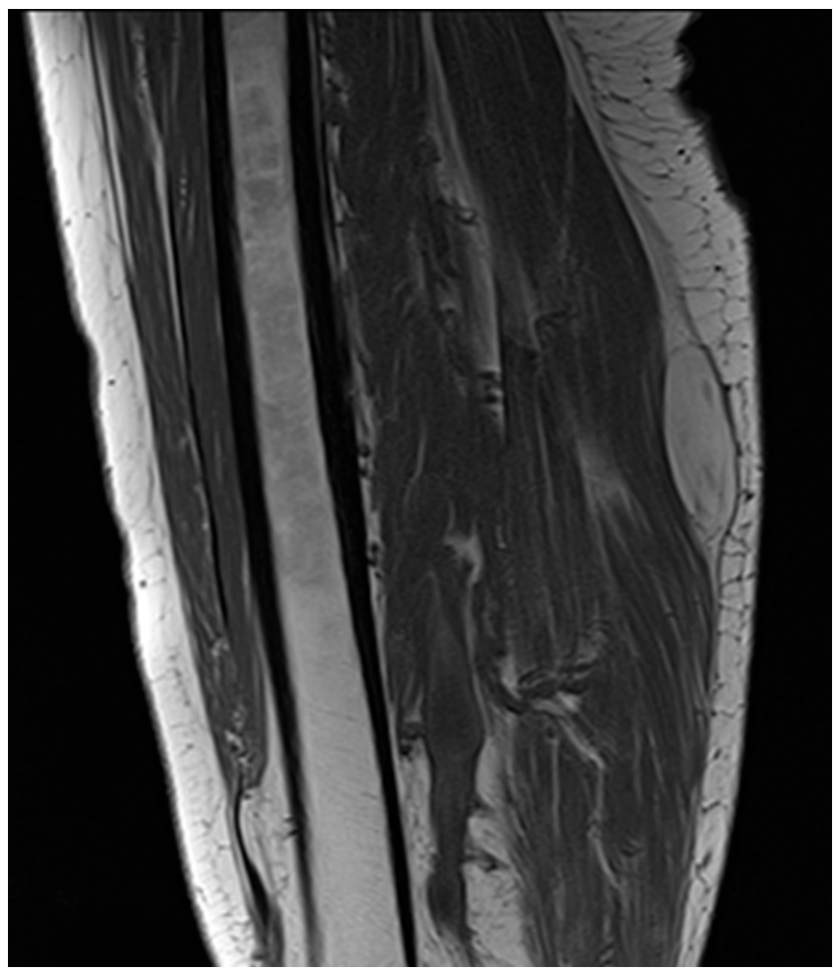

(d)

Figure 4. A 79-year-old woman operated on for a high-grade liposarcoma. At follow-up, both US (a) and MRI (b-d) detected a solid nodule in the posterior aspect of the thigh with prevalent fatty content near the surgical area. Both US and MRI were reported as consistent with recurrence; however, pathology did not show any malignant tissue after surgery.
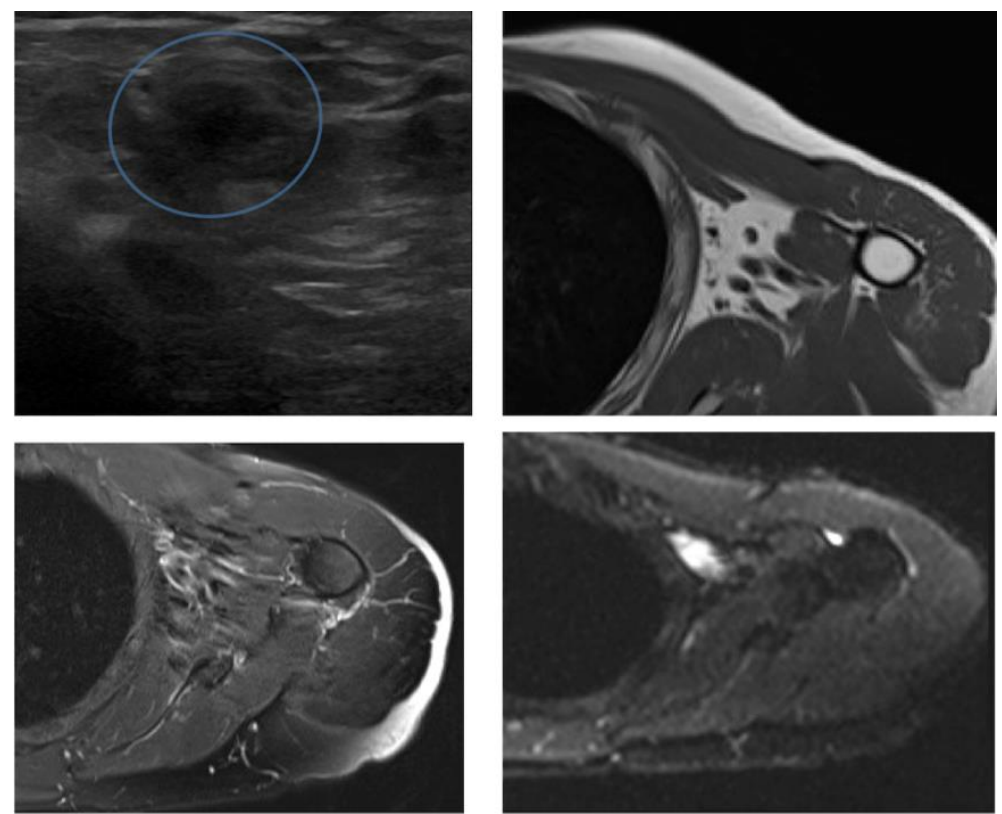

Figure 5. A 71-year-old man with intermediate-grade leiomyosarcoma of the proximal arm. US was reported as positive for recurrence due to the detection of a pseudomass/irregularity of the superficial tissues of the proximal arm (circle). MRI did not show any mass. Follow-up did not show any recurrence. 


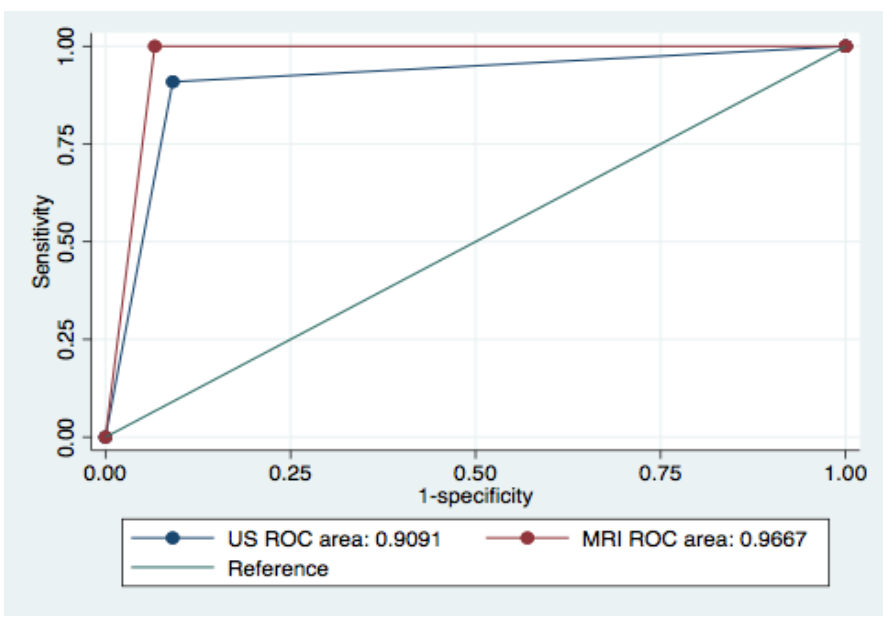

Figure 6. ROC analysis of US and MR imaging follow-up.

Table 2. (a) Summary of diagnostic accuracy parameters for US and MRI. (b) $2 \times 2$ cross-tabulation with number of positive and negative scans. $\mathrm{TP}=$ true positive, $\mathrm{TN}$, true negative, $\mathrm{FN}=$ false negative, $\mathrm{FP}=$ false positive. $\left(^{*}\right)$ These values are dependent on disease prevalence.

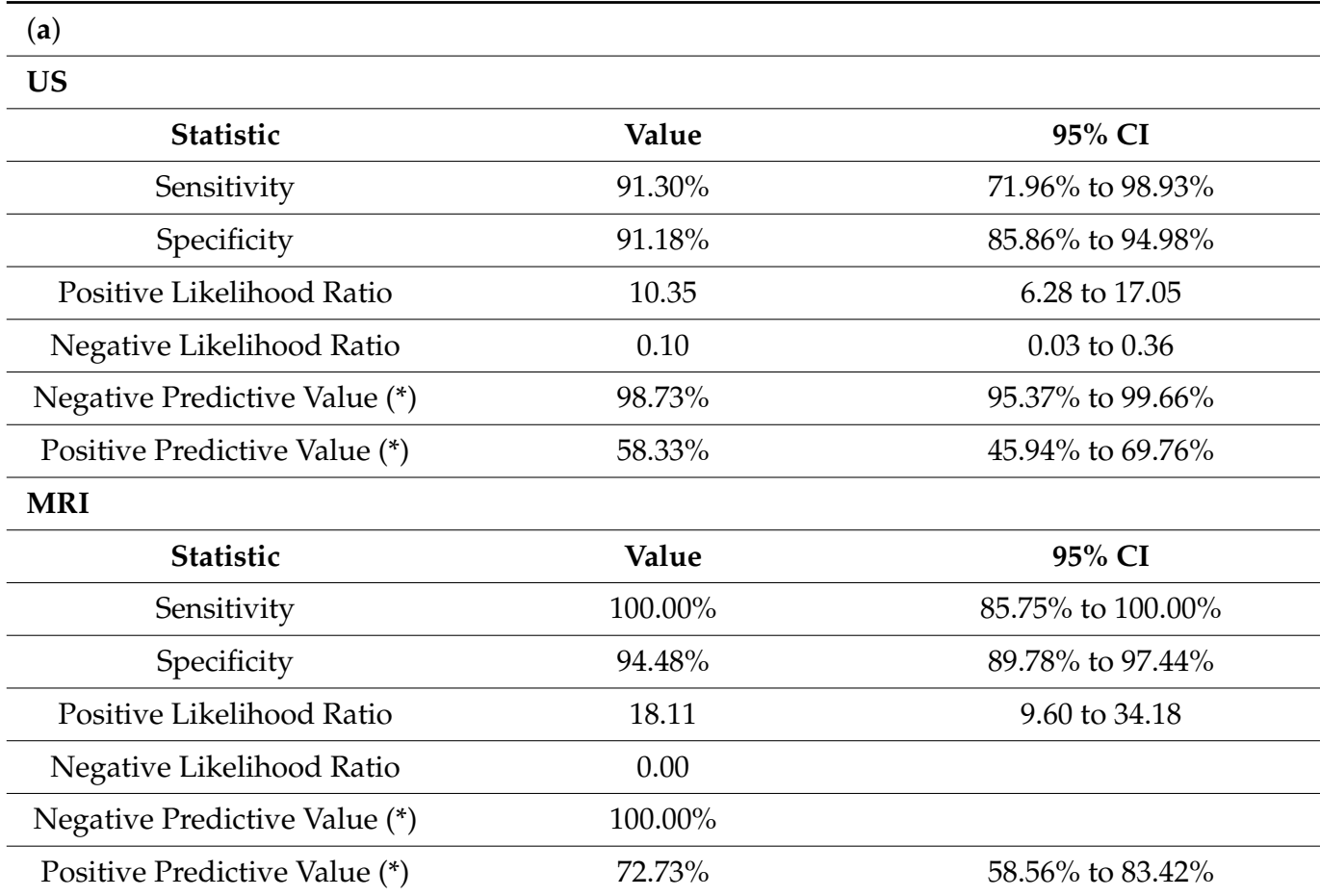

(b) $2 \times 2$ cross-tabulation of US vs. MRI. $X^{2} p$-value: 0.0766 .

\begin{tabular}{ccc}
\hline & US + & US - \\
\hline MR + & 31 & 3 \\
\hline MR - & 0 & 198 \\
\hline
\end{tabular}

NB: No true positive and false positive included.

\begin{tabular}{ccc}
\hline US & MRI & \\
\hline 21 & 24 & TP \\
\hline 194 & 199 & TN \\
\hline 2 & 0 & FN \\
\hline 15 & 9 & FP \\
\hline
\end{tabular}




\section{Discussion}

According to recent guidelines on STT imaging and follow-up, we tried to fill the gap regarding the need to have prospective studies formally comparing US and MRI in the follow-up of patients operated for localized intermediate or high-risk soft tissue tumors of the extremities $[2,10,18]$. We designed a comparative study where the two imaging techniques used more often to rule out LR, US, and MRI, were compared in the same patient. The study design included independent radiological evaluation of US and MRI to overcome the need for a randomized trial that is unfeasible for ethical reasons, and to reduce the number of patients to be included as already performed in other imaging studies [21-25]. To increase the reliability of this approach and reduce biases, we allowed a $<7$ days of time frame between US and MRI, limiting as much as possible to have both US and MRI in the same session. On the other hand, we did not choose a randomized comparative trial to avoid offering patients a potentially worst radiological technique than the alternative. In other words, this study design allowed patients to receive the best imaging follow-up available so far. Results of this trial show that both US and MRI are highly sensitive in detecting LRs in this group of patients with localized disease, although overall accuracy of MRI resulted slightly higher: AUC for US was 0.909 (95\% C.I. $=0.832$ to 0.981 ) for US and AUC for MRI 0.966 ( $95 \%$ C.I. $=0.939$ to 0.989) for MRI. When comparing US and MRI performances, there was no formal statistical difference between the two techniques if $X^{2}$ statistics was used considering the 232 events that were included in the analysis. The present study included 37 patients: adding more patients and follow-up events could have increased the chances of having a statistically significant result in favor of MR, but it is not sure that the result would have been clinically meaningful. The results of this prospective study seem to confirm the results obtained in a retrospective analysis focused on the performance of ultrasound [13]. Indeed, US resulted in being highly specific for LRs detection with few false positives due to the presence of scarring and granulation tissue that resembled a mass lesion. Using ultrasound probes with low frequencies, $5 \mathrm{MHz}$ to $7.5 \mathrm{MHz}$, which are different from up-to-date broadband linear array probes and selecting a few patients Choi et al., in 1991, stated that the accuracy of US for the detection of local recurrence was similar to that of MRI. On 26 patients, they reported $100 \%$ sensitivity and $79 \%$ specificity for US and $83 \%$ and $93 \%$, respectively, for MRI [26,27]. In 1993, an Italian research group from the National Institute for Cancer Research of Genova led by Pino et al. [27], evaluating the same number of patients of Choi et al. [26], reported a sensitivity of $87 \%$ for US, compared with $69.6 \%$ for computed tomography for lesions up to $5 \mathrm{~mm}$. Arya et al. [28], in 2000, evaluated with US 50 patients operated for sarcoma surgery finding 26 recurrences, 18 non-recurrences, 4 benign diseases, and 2 indeterminate lesions reporting a sensitivity for US of $92.3 \%$ and a specificity of $94.4 \%$.

US has obvious advantages of cost efficiency compared to MRI, but US, especially in the post-surgical setting, has the advantage of not being subject to artifacts from metallic hardware and to potentially guide drainage (and obviously biopsy) [29]. Our surveillance protocol can be considered intense, and regarding the use of MRI or US, we cannot affirm that one technique is clearly better than the other; however, the overall performance of MRI resulted in being slightly better than that of US. In the present study, US resulted in being less effective in detecting deep lesions, for example, at the popliteal fossa for lesions containing fat. This is one of the few studies with prospective data helping to address an unmet need in clinical follow-up of patients with STT of the extremities [2,10,12,18]. Differently from the work by Singer et al. [30], in our study, the reference standard was histology, surgery, or follow-up for both MRI and US. Singer et al. [30] considered a subsequent MRI as a possible reference standard for US, therefore, introducing a bias related to the reference standard.

Compared to the study of Singer et al. [30] and Tagliafico et al. [11,13], our results in terms of accuracy could be considered overlapping in spite of slightly different study designs. In this study, AUC for US was 0.909 (91\%), whereas, in the study of Singer 
et al. [30], the accuracy was 92.6\%, and the AUC for MRI was 0.966 (96\%) compared to $97.6 \%$ in the study of Singer et al. [30].

This study, although it is one of the few prospective studies directly comparing US and MRI in the same patient with the surgical and histological reference standards, has some limitations. Due to the relative rarity of STT and to the necessity of having strict protocol adherence (see inclusion criteria), the study cohort could be considered moderate in number. However, this study cohort is comparable to previous studies, and it was evaluated with a better study design $[13,30]$.

US is known to have reduced performance in deeply located lesions, and it is normally considered operator dependent. Indeed, the STTs analyzed in this study are mostly of the upper limb. In the case of a deeply located lower limb tumor, it is possible that the referring physician did not send the patients for US or MRI in this center due to the known limitations of US in deeply located lesions. In this study, radiologists were highly experienced in US usage, and we do not know if these US performances are clearly reproducible at other institutions and to what extent. However, there are many efforts in the scientific community to develop guidelines and to standardize US usage among different specialists to have reliable and repeatable US examinations [10,12,31-34]. Against the usage of MR, a study by there is a study by Labarre D et al. [35] stated that the systematic use of MR was not useful to detect asymptomatic local recurrences. However, MR is still considered the technique of choice for follow-up in spite of suitable and comparable performances of US. We would like to clarify that it is extremely probable that US performed in non-specialized centers by different operators would not be able to achieve these levels of reliability compared to MRI. Further larger prospective studies are needed to further clarify this issue.

In conclusion, this prospective study compared prospectively and with independent reading US and MRI in patients with localized intermediate or high-risk soft tissue tumors of the extremities. Each of these tests detected local recurrences with suitable accuracy. Incorporation of US in surveillance algorithms of patients with STT of the extremity STS have several advantages (even psychological) and could be discussed in guidelines, but the level of reliability of MRI is extremely high. It has still to be demonstrated that US performed in non-specialized centers by different operators is not inferior to MRI.

Author Contributions: The scientific guarantor of this publication is A.S.T. A.S.T.: study design and scientific direction, data analysis and interpretation, literature searches, manuscript writing, and revision. F.R., B.B., C.M., B.S., A.S. and N.S.: screen-reading, data collection. F.R., B.B., C.M., B.S. and N.S.: data collection and overall supervision. A.S.T.: study design and scientific direction, data interpretation, manuscript writing, and revision. A.S.T.: statistical supervision. Agreement to be accountable for all aspects of the work in ensuring that questions related to the accuracy or integrity of any part of the work are appropriately investigated and resolved: all authors. Final approval of the version to be published: all authors. All authors have read and agreed to the published version of the manuscript.

Funding: The project is partially supported by the AIUM (American Institute of Ultrasound in Medicine) Endowment for Education and Research (EER) with a grant to Alberto Tagliafico. The study is partially supported by the University of Genova with a grant (PRA 2014) to Carlo Martinoli. This study is also supported by ESSR (European Society of Musculoskeletal Radiology) through one committee, the Research (Chair 2016 Alberto Tagliafico), and two sub-committees: Ultrasound (Chair 2016 Luca Sconfienza) and Tumor (Chair 2016 MarcAndrè Weber).

Institutional Review Board Statement: Approval from the Regione Liguria institutional review board was obtained accordingly with the Italian laws (304REG2015).

Informed Consent Statement: Informed consent was obtained from all subjects involved in the study. Written informed consent has been obtained from the patient(s) to publish this paper.

Data Availability Statement: The data presented in this study are available on request from the corresponding author.

Conflicts of Interest: The authors declare no conflict of interest. 


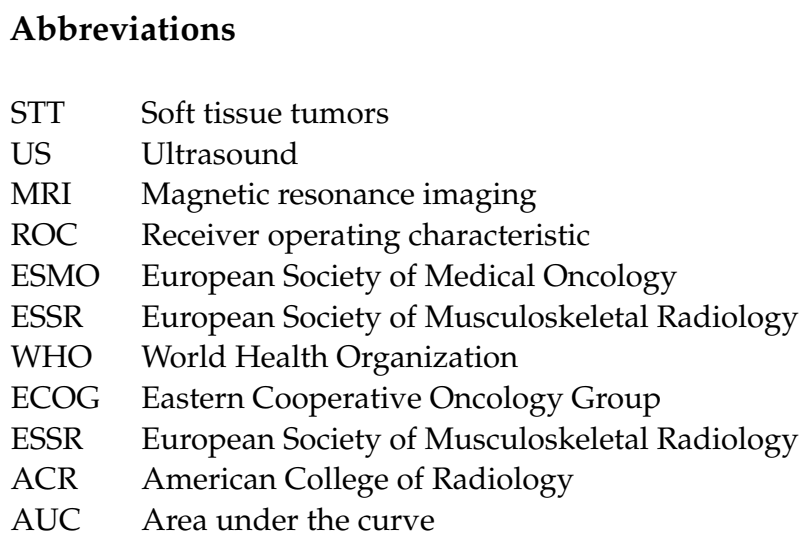

\section{References}

1. Gronchi, A.; Vullo, S.L.; Colombo, C.; Collini, P.; Stacchiotti, S.; Mariani, L.; Fiore, M.; Casali, P.G. Extremity Soft Tissue Sarcoma in a Series of Patients Treated at a Single Institution: Local control directly impacts survival. Ann. Surg. 2010, 251, 506-511. [CrossRef] [PubMed]

2. Casali, P.G.; Abecassis, N.; Bauer, S.; Biagini, R.; Bielack, S.; Bonvalot, S.; Boukovinas, I.; Bovee, J.V.M.G.; Brodowicz, T.; Broto, J.; et al. Soft tissue and visceral sarcomas: ESMO-EURACAN Clinical Practice Guidelines for diagnosis, treatment and follow-up. Ann. Oncol. 2018, 29 (Suppl. S4), iv51-iv67. [CrossRef]

3. Eilber, F.C.; Rosen, G.; Nelson, S.D.; Selch, M.; Dorey, F.; Eckardt, J.; Eilber, F.R. High-Grade Extremity Soft Tissue Sarcomas: Factors predictive of local recurrence and its effect on morbidity and mortality. Ann. Surg. 2003, 237, 218-226. [CrossRef] [PubMed]

4. Zagars, G.K.; Ballo, M.; Pisters, P.W.; Pollock, R.E.; Patel, S.R.; Benjamin, R.S. Prognostic factors for disease-specific survival after first relapse of soft-tissue sarcoma: Analysis of 402 patients with disease relapse after initial conservative surgery and radiotherapy. Int. J. Radiat. Oncol. Biol. Phys. 2003, 57, 739-747. [CrossRef]

5. Nakamura, T.; Abudu, A.; Murata, H.; Grimer, R.; Carter, S.; Tillman, R.; Jeys, L. Oncological outcome of patients with deeply located soft tissue sarcoma of the pelvis: A follow up study at minimum 5 years after diagnosis. Eur. J. Surg. Oncol. J. Eur. Soc. Surg. Oncol. Br. Assoc. Surg. Oncol. 2013, 39, 1030-1035. [CrossRef]

6. Italiano, A.; Le Cesne, A.; Mendiboure, J.; Blay, J.-Y.; Piperno-Neumann, S.; Chevreau, C.; Delcambre, C.; Penel, N.; Terrier, P.; Ranchere-Vince, D.; et al. Prognostic factors and impact of adjuvant treatments on local and metastatic relapse of soft-tissue sarcoma patients in the competing risks setting. Cancer 2014, 120, 3361-3369. [CrossRef]

7. Gamboa, A.C.; Gronchi, A.; Cardona, K. Soft-tissue sarcoma in adults: An update on the current state of histiotype-specific management in an era of personalized medicine. CA A Cancer J. Clin. 2020, 70, 200-229. [CrossRef] [PubMed]

8. Correa, R.; Goméz-Millan, J.; Lobato, M.; Fernandez, A.; Ordoñez, R.; Castro, C.; Lupiañez, Y.; Medina, J.A. Radiotherapy in soft-tissue sarcoma of the extremities. Clin. Transl. Oncol. Off. Publ. Fed. Span. Oncol. Soc. Natl. Cancer Inst. Mex. 2018, 20, 1127-1135. [CrossRef]

9. $\quad$ Chou, Y.-S.; Liu, C.-Y.; Chen, W.-M.; Chen, T.-H.; Chen, P.C.-H.; Wu, H.-T.H.; Chiou, H.-J.; Shiau, C.-Y.; Wu, Y.-C.; Liu, C.-L.; et al. Follow-up after primary treatment of soft tissue sarcoma of extremities: Impact of frequency of follow-up imaging on disease-specific survival. J. Surg. Oncol. 2012, 106, 155-161. [CrossRef]

10. Noebauer-Huhmann, I.M.; Weber, M.-A.; Lalam, R.K.; Trattnig, S.; Bohndorf, K.; Vanhoenacker, F.; Tagliafico, A.; Van Rijswijk, C.; Vilanova, J.C.; Afonso, P.D.; et al. Soft Tissue Tumors in Adults: ESSR-Approved Guidelines for Diagnostic Imaging. Semin. Musculoskelet. Radiol. 2015, 19, e1. Erratum in Semin. Musculoskelet. Radiol. 2015, 19, 475-482.

11. Tagliafico, A.S.; Bignotti, B.; Rossi, F.; Valdora, F.; Martinoli, C. Local recurrence of soft tissue sarcoma: A radiomic analysis. Radiol. Oncol. 2019, 53, 300-306. [CrossRef] [PubMed]

12. Weiss, S.; Korthaus, A.; Baumann, N.; Yamamura, J.; Spiro, A.S.; Lübke, A.M.; Frosch, K.-H.; Schlickewei, C.; Priemel, M. Musculoskeletal Soft-Tissue Sarcoma: Quality Assessment of Initial MRI Reports Shows Frequent Deviation from ESSR Guidelines. Diagnostics 2021, 11, 695. [CrossRef] [PubMed]

13. Tagliafico, A.; Truini, M.; Spina, B.; Cambiaso, P.; Zaottini, F.; Bignotti, B.; Calabrese, M.; Derchi, L.E.; Martinoli, C. Follow-up of recurrences of limb soft tissue sarcomas in patients with localized disease: Performance of ultrasound. Eur. Radiol. 2015, 25, 2764-2770. [CrossRef] [PubMed]

14. Tagliafico, A.S.; Isaac, A.; Bignotti, B.; Rossi, F.; Zaottini, F.; Martinoli, C. Nerve Tumors: What the MSK Radiologist Should Know. Semin. Musculoskelet. Radiol. 2019, 23, 76-84. [CrossRef]

15. Bull, S.B. Sample Size and Power Determination for a Binary Outcome and an Ordinal Exposure when Logistic Regression Analysis Is Planned. Am. J. Epidemiol. 1993, 137, 676-684. [CrossRef]

16. Erturk, S.M. Retrospective Power Analysis: When? Radiology 2005, 237, 743-744. [CrossRef]

17. Maretty-Nielsen, K. Prognostic factors in soft tissue sarcoma. Dan. Med. J. 2014, 61, B4957.

18. Patel, S.R.; Zagars, G.K.; Pisters, P.W. The follow-up of adult soft-tissue sarcomas. Semin. Oncol. 2003, 30, 413-416. [CrossRef] 
19. Hagedorn, K.N.; Hayatghaibi, S.E.; Levine, M.H.; Orth, R.C. Cost Comparison of Ultrasound Versus MRI to Diagnose Adolescent Female Patients Presenting with Acute Abdominal/Pelvic Pain Using Time-Driven Activity-Based Costing. Acad. Radiol. 2019, 26, 1618-1624. [CrossRef]

20. Xu, H.; Zhang, Y.; Zhang, H.; Wang, C.; Mao, P. Comparison of the clinical effectiveness of US grading scoring system vs MRI in the diagnosis of early rheumatoid arthritis (RA). J. Orthop. Surg. Res. 2017, 12, 152. [CrossRef]

21. Aghdassi, A.A.; Schauer, B.; Duscha, D.; Ittermann, T.; Pickartz, T.; Budde, C.; Simon, P.; Moskwa, P.; Kromrey, M.L.; Bülow, R.; et al. Comparability of size measurements of the pancreas in magnetic resonance imaging and transabdominal ultrasound. Clin. Anat. 2020, 33, 431-439. [CrossRef] [PubMed]

22. Cantisani, V.; Ricci, P.; Erturk, M.; Pagliara, E.; Drudi, F.M.; Calliada, F.; Mortele, K.; D’Ambrosio, U.; Marigliano, C.; Catalano, C.; et al. Detection of Hepatic Metastases from Colorectal Cancer: Prospective Evaluation of Gray Scale US Versus SonoVue ${ }^{\circledR L}$ Low Mechanical Index Real Time-Enhanced US as Compared with Multidetector-CT or Gd-BOPTA-MRI. Ultraschall Med. 2010, 31, 500-505. [CrossRef] [PubMed]

23. Ha, S.M.; Cha, J.H.; Shin, H.J.; Chae, E.Y.; Choi, W.J.; Kim, H.H. Mammography, US, and MRI to Assess Outcomes of Invasive Breast Cancer with Extensive Intraductal Component: A Matched Cohort Study. Radiology 2019, 292, 299-308. [CrossRef] [PubMed]

24. Hakozaki, Y.; Matsushima, H.; Kumagai, J.; Murata, T.; Masuda, T.; Hirai, Y.; Oda, M.; Kawauchi, N.; Yokoyama, M.; Homma, Y. A prospective study of magnetic resonance imaging and ultrasonography (MRI/US)-fusion targeted biopsy and concurrent systematic transperineal biopsy with the average of 18-cores to detect clinically significant prostate cancer. BMC Urol. 2017, 17, 117. [CrossRef]

25. Kim, H.R.; Jung, H.K.; Ko, K.H.; Kim, S.J.; Lee, K.S. Mammography, US, and MRI for Preoperative Prediction of Extensive Intraductal Component of Invasive Breast Cancer: Interobserver Variability and Performances. Clin. Breast Cancer 2016, 16, 305-311. [CrossRef]

26. Choi, H.; Varma, D.G.; Fornage, B.D.; Kim, E.E.; Johnston, D.A. Soft-tissue sarcoma: MR imaging vs sonography for detection of local recurrence after surgery. AJR Am. J. Roentgenol. 1991, 157, 353-358. [CrossRef]

27. Pino, G.; Conzi, G.F.; Murolo, C.; Schenone, F.; Magliani, L.; Imperiale, A.; Dato, G.; Panetta, M.; Toma, S. Sonographic evaluation of local recurrences of soft tissue sarcomas. J. Ultrasound Med. Off. J. Am. Inst. Ultrasound Med. 1993, 12, 23-26. [CrossRef]

28. Arya, S.; Nagarkatti, D.G.; Dudhat, S.B.; Nadkarni, K.S.; Joshi, M.S.; Shinde, S.R. Soft Tissue Sarcomas: Ultrasonographic Evaluation of Local Recurrences. Clin. Radiol. 2000, 55, 193-197. [CrossRef]

29. Gómez, M.P.A.; Errani, C.; Lalam, R.; Nikodinovska, V.V.; Fanti, S.; Tagliafico, A.S.; Sconfienza, L.M.; Bazzocchi, A. The Role of Ultrasound in the Diagnosis of Soft Tissue Tumors. Semin. Musculoskelet. Radiol. 2020, 24, 135-155. [CrossRef]

30. Singer, A.D.; Wong, P.; Umpierrez, M.; Reimer, N.; Gonzalez, F.; Reiter, D.; Cardona, K. The accuracy of a novel sonographic scanning and reporting protocol to survey for soft tissue sarcoma local recurrence. Skelet. Radiol. 2020, 49, 2039-2049. [CrossRef]

31. Sconfienza, L.M.; Albano, D.; Allen, G.; Bazzocchi, A.; Bignotti, B.; Chianca, V.; De Castro, F.F.; Drakonaki, E.E.; Gallardo, E.; Gielen, J.; et al. Clinical indications for musculoskeletal ultrasound updated in 2017 by European Society of Musculoskeletal Radiology (ESSR) consensus. Eur. Radiol. 2018, 28, 5338-5351. [CrossRef] [PubMed]

32. Tagliafico, A.S.; Wilson, D.; Sconfienza, L.M. Encouraging MSK imaging research towards clinical impact is a necessity: Opinion paper of the European Society of Musculoskeletal Radiology (ESSR). Eur. Radiol. 2019, 29, 3410-3413. [CrossRef]

33. Barnett, S.B.; ter Haar, G.R.; Ziskin, M.C.; Rott, H.-D.; Duck, F.A.; Maeda, K. International recommendations and guidelines for the safe use of diagnostic ultrasound in medicine. Ultrasound Med. Biol. 2000, 26, 355-366. [CrossRef]

34. Dietrich, C.F.; Abramowicz, J.S.; Chammas, M.C.; Chou, Y.-H.; Condous, G.; Kim, S.H.; Nolsøe, C.P.; Vinayak, S.; Jenssen, C. World Federation for Ultrasound in Medicine and Biology (WFUMB) Policy Document Development Strategy-Clinical Practice Guidelines, Position Statements and Technological Reviews (on behalf of the WFUMB publication committee and Executive Bureau). Ultrasound Med. Biol. 2021, 47, 2779-2781. [CrossRef] [PubMed]

35. Labarre, D.; Aziza, R.; Filleron, T.; Delannes, M.; Delaunay, F.; Marques, B.; Ferron, G.; Chevreau, C. Detection of local recurrences of limb soft tissue sarcomas: Is magnetic resonance imaging (MRI) relevant? Eur. J. Radiol. 2009, 72, 50-53. [CrossRef] [PubMed] 